\title{
Linked-color imaging combined with the NICE classification system for optical diagnosis of colon polyps: new image-enhanced endoscopic technology for pathological prediction
}

\author{
This article was published in the following Dove Press journal: \\ Therapeutics and Clinical Risk Management \\ 3 October 2017 \\ Number of times this article has been viewed
}

\author{
Chi-Huan $\mathrm{Wu}^{1,2}$ \\ Tsung-Hsing Chen ${ }^{1-3}$ \\ Chen-Ming $\mathrm{Hsu}^{1,2}$ \\ Ming-Yao Su ${ }^{1,2}$ \\ Cheng-Tang Chiu', ${ }^{1,2}$ \\ Ren-Chin $\mathrm{Wu}^{4}$ \\ Cheng-Chou Lai ${ }^{5}$ \\ 'Department of Gastroenterology \\ and Hepatology, Linkou Medical \\ Center, Chang Gung Memorial \\ Hospital, Taoyuan, ${ }^{2}$ Chang Gung \\ University, College of Medicine, \\ Taoyuan, ${ }^{3} \mathrm{Graduate}$ Institute of \\ Clinical Medical Sciences, Chang Gung \\ University, Taoyuan, ${ }^{4}$ Department of \\ Pathology, Linkou Medical Center, \\ Chang Gung Memorial Hospital, \\ Taoyuan, ${ }^{5}$ Department of Colon and \\ Rectal Surgery, Linkou Medical Center, \\ Chang Gung Memorial Hospital, \\ Taoyuan, Taiwan
}

Introduction: Linked-color imaging (LCI) is a recently developed system used in endoscopy. It creates clear and bright endoscopic images using short-wavelength, narrow-band laser light combined with white laser light. The illuminating light and signal processing emphasize slight color differences in abnormal regions that approximate the normal color of the mucosa. As a result, regions initially appearing red become a deeper shade of red, while regions originally appearing white become brighter, yet with natural tones. This process facilitates recognition of slight differences in the color of the mucosa and clarifies the boundaries of the mucosal pit.

Aim: To determine whether LCI of the colon can improve the correlation between endoscopic findings and pathological diagnosis.

Methods: Consecutive patients who underwent colonoscopy requiring polypectomy or removal by biopsy forceps if possible were recruited. Probable polyp histology was assessed by two endoscopists using the Narrow-band imaging International Colorectal Endoscopic (NICE) classification and LCI data. All detected polyps were sent to the pathology department for pathological diagnosis by two pathologists.

Results: In total, 94 polyps were found in 43 patients. The sensitivity, specificity, positive predictive value, and negative predictive value for neoplastic lesion prediction (NICE type2/3) were $96.5 \%, 83.8 \%, 90.2 \%$, and $93.9 \%$, respectively.

Conclusion: LCI combined with the NICE classification system is a powerful tool for predicting probable histology of colon polyps.

Keywords: linked-color imaging, NICE classification, colon polyps, histology, sensitivity, specificity

\section{Introduction}

Colonoscopy to survey polyps requiring polypectomy is considered to be the most effective method of improving colorectal cancer-related morbidity and mortality. ${ }^{1}$ There are several types of polyps, which can be divided into nonneoplastic (ie, hyperplastic) and neoplastic (ie, adenomatous) lesion types. The typical pathologic image of nonneoplastic lesions and neoplastic lesions are shown in Figure 1. Removal of all adenomatous polyps during colonoscopy has been the most effective method of preventing colon cancer. Pathological examination, however, is the most accurate method of characterizing and diagnosing polyps. Endoscopists are currently searching for more effective methods of distinguishing different types of polyps during colonoscopy. This has prompted the development of several types of imaging techniques and colonoscopy devices to
Correspondence: Tsung-Hsing Chen Department of Gastroenterology and Hepatology, Linkou Medical Center, Chang Gung Memorial Hospital, 5 Fu-Hsin Street, Queishan, Taoyuan County 333, Taiwan

Tel +88633281200 ext 8102

Fax +88633272236

Email itochenyu@gmail.com 

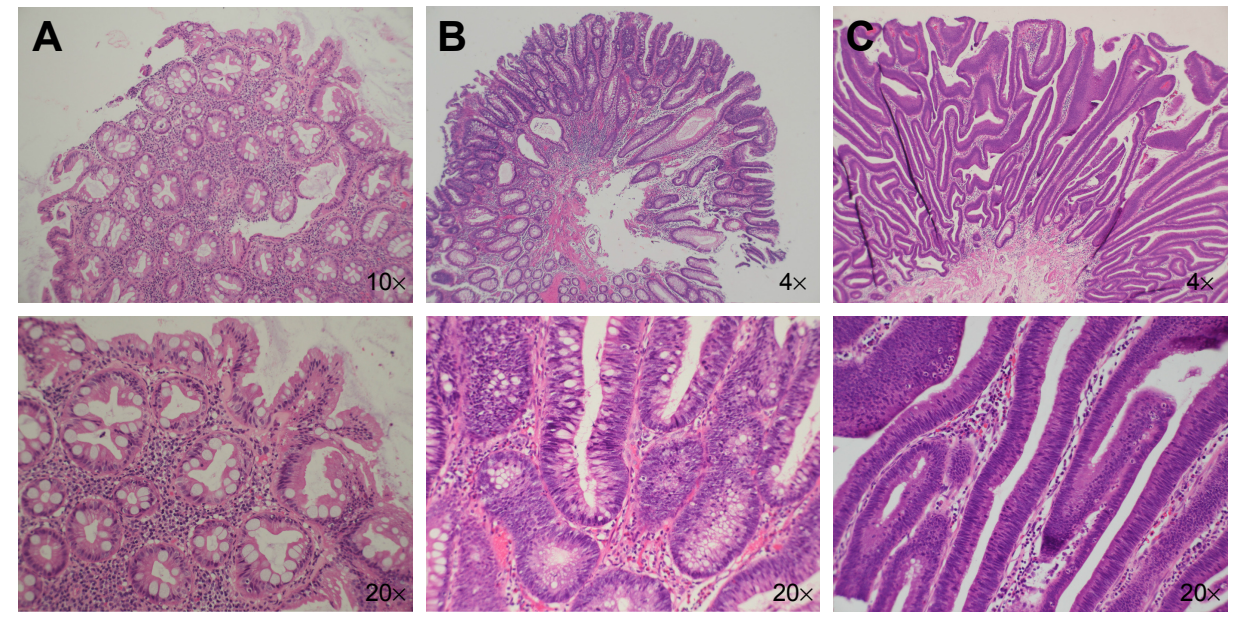

Figure I Photomicrographs (hematoxylin \& eosin stain) of different types of colon polyps.

Notes: Histologically, a hyperplastic polyp shows epithelial hyperplasia characterized by a serrated appearance without nuclear atypia (A); a tubular adenoma contains elongated crypts lined by columnar epithelial cells with nuclear atypia, including nuclear elongation, pleomorphism, and hyperchromasia (B); a villous adenoma is characterized by a villiform architecture lined by columnar epithelial cells with nuclear atypia similar to that seen in a tubular adenoma (C). Nx: picture taken with $\mathrm{Nx}$ objective lens.

enhance surface and vascular patterns to improve diagnostic yield. Narrow-band imaging (NBI), image-enhanced endoscopy (i-scan, Pentax, Montvale, NJ, USA), and blue laser imaging and linked-color imaging (LCI) systems are currently in use. In addition to these new technologies, there are also several classification systems used for pathological prediction of colon polyps, including the Hiroshima, Sano, Showa, and the NBI International Colorectal Endoscopic (NICE) classifications. Of these methods, the NICE classification system is the most convenient for daily practice because it can be applied using colonoscopes with or without optical (ie, zoom) magnification capability. The NICE classification was first introduced by The Colon Tumor NBI Interest Group in 2009. ${ }^{2}$ The NICE classification system was the first NBI classification that did not require magnifying endoscopy; this has facilitated its widespread use all over the world. Although the classification scheme was initially designed for use with
NBI, it has also demonstrated utility in other image-enhanced endoscopy methods, such as i-scan. ${ }^{3}$

Recently, a new image-enhanced endoscopy system, known as LCI, has been developed that provides clear and bright endoscopic images using short-wavelength, narrowband laser light combined with white laser light. The LCI system enables red areas to appear redder and white areas to appear brighter. Thus, it is an appropriate tool for recognizing color differences in the mucosa because it enhances clarity and recognition of the mucosal pit, which in turn facilitates pathological prediction of colon polyps (Figure 2). However, there have been no studies investigating the use of the NICE classification with data obtained using LCI. Accordingly, the aim of our study was to determine the feasibility of using the NICE classification combined with data obtained using an LCI system without magnification to predict colon polyp pathology.
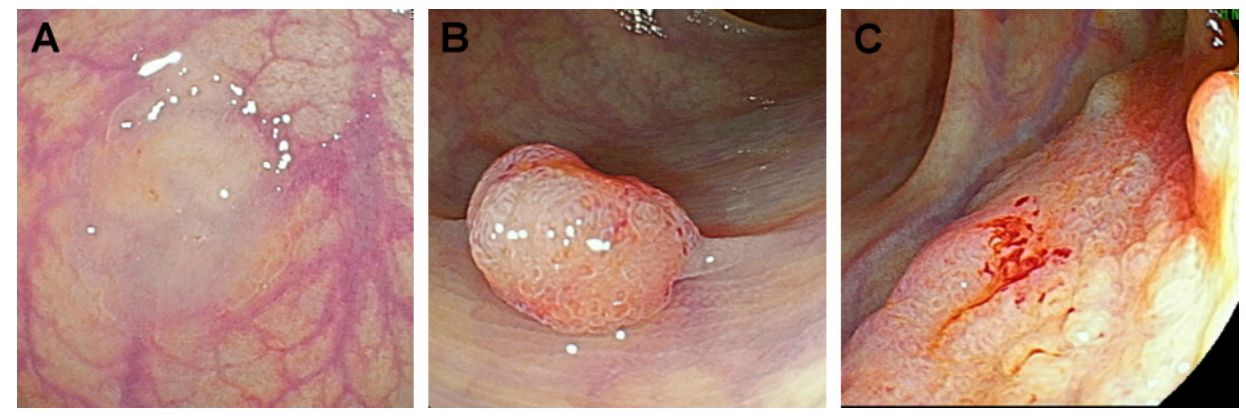

Figure 2 Endoscopic findings using LCl.

Notes: Lesion classified as NICE type I (A); lesion classified as NICE type 2 (B); lesion classified as NICE type 3 (C).

Abbreviations: LCI, linked-color imaging; NICE, Narrow-band imaging International Colorectal Endoscopic. 


\section{Methods}

\section{Patients}

Chang Gung Memorial Hospital's Institutional Review Board Committee approved the study protocol (Code of IRB: 201600789B0). This retrospective study recruited consecutive patients who underwent colonoscopy requiring polypectomy or endoscopic biopsy based on LCI findings. The institutional review board waived patient written informed consent due to deidentified medical records.

\section{Colonoscopy procedure}

For bowel preparation, patients ingested 1.5-2 L of polyethylene glycol or $90 \mathrm{~mL}$ of a split-dose phospho-soda solution (Fleet; CB Fleet Co, Lynchburg, VA, USA) before the procedure. Two colonoscopists performed all colonoscopy procedures up to the cecum using high-resolution endoscopy (EC-L590ZW/L; Fujifilm, Tokyo, Japan) and a laser light source (Lasereo; Fujifilm).

\section{Endoscopic diagnosis using the NICE classification}

All lesions were initially detected using conventional viewing methods and were then examined using the LCI system without magnification to evaluate endoscopic surface features. All lesions were subsequently classified into one of three types based on the NICE classification (Table 1).

\section{Clinicopathological evaluation}

Medical records for lesion size, shape (according to Paris classification), ${ }^{4} \mathrm{NICE}$ classification category, and pathological diagnosis were reviewed. Two pathologists blinded to the clinical information diagnosed all cases together with consistency consent. Using this information, the relationship between LCI data, NICE classification, and the histopathological findings were examined.

\section{Results}

A total of 94 polyps were detected in 43 patients ( 32 male, 11 female; mean age 54.2 years [range $42-75$ years]). The mean polyp size was $17 \mathrm{~mm}$ (range 2-25 mm). Seven polyps were pedunculated, 63 were sessile, and 24 were flat. Thirtythree (35\%) polyps were classified as NICE type 1 and, based on pathology, 31 (93.9\%) of these were hyperplastic and sessile serrated adenomas/polyps, and two (6.1\%) were low-grade adenomas (Table 2). Fifty-nine polyps were classified as NICE type 2, $49(83.1 \%)$ of these were lowgrade adenomas, six $(10.1 \%)$ were hyperplastic or sessile serrated adenomas/polyps, and four (6.7\%) were high-grade adenomas. The remaining two polyps included a high-grade adenoma and an invasive carcinoma, both of which exhibited endoscopic features classified as NICE type 3 (Table 3).

Histologically, colorectal polyps are classified as either neoplastic (NICE type 2/3) or nonneoplastic (NICE type 1). The endoscopist in this study correctly predicted neoplastic histology in 55 of 61 polyps and nonneoplastic histology in 31 of 33 polyps. Therefore, the sensitivity, specificity, positive predictive value (PPV), and negative predictive value (NPV) for neoplastic lesion prediction were $96.5 \%, 83.8 \%$, $90.2 \%$, and $93.9 \%$, respectively (Table 4 ).

\section{Discussion}

The adenoma-carcinoma sequence was described by Morson $^{5}$ in 1974. Based on this study, endoscopists worldwide recognized that removing all adenomatous polyps is among the most important goals of colonoscopy. However, the "resect and discard" policy is currently advocated, ${ }^{6,7}$ not only to reduce the risk for bleeding or perforation during the polyp removal procedure but also to eliminate the associated costs of pathological examination. For this reason, various image-enhanced endoscopy systems are available in clinical practice to improve detection and histological prediction

Table I NICE classification summary ${ }^{\mathrm{a}}$

\begin{tabular}{|c|c|c|c|}
\hline Characteristic & Type I & Type $2^{\text {b }}$ & Type 3 \\
\hline Color & Same or lighter than background & $\begin{array}{l}\text { Browner relative to background (verify } \\
\text { color arises from vessels) }\end{array}$ & $\begin{array}{l}\text { Brown to dark brown relative to } \\
\text { background; sometimes patchy whiter areas }\end{array}$ \\
\hline Vessels & $\begin{array}{l}\text { None, or isolated, lacy vessels coursing } \\
\text { across the lesion may be present }\end{array}$ & $\begin{array}{l}\text { Brown vessels surrounding white } \\
\text { structures }^{c}\end{array}$ & Area(s) of disrupted or missing vessels \\
\hline Surface pattern & $\begin{array}{l}\text { Dark or white spots of uniform size, } \\
\text { or homogeneous absence of pattern }\end{array}$ & $\begin{array}{l}\text { Oval, tubular, or branched white } \\
\text { structures surrounded by brown vessels }\end{array}$ & Amorphous or absent \\
\hline Most likely pathology & Hyperplastic & Adenoma & Deep submucosal invasive cancer \\
\hline
\end{tabular}

Notes: a Can be applied using colonoscopes with or without optical (zoom) magnification capability; ${ }^{\text {T}}$ Type 2 consists of Vienna classification types 3 , 4 , and superficial 5 (all adenomas with either low- or high-grade dysplasia, or with superficial submucosal carcinoma). The presence of high-grade dysplasia or superficial submucosal carcinoma may be suggested by an irregular vessel or surface pattern and is often associated with atypical morphology (eg, depressed area); 'these structures (regular or irregular) may represent the pits and the epithelium of the crypt opening.

Abbreviation: NICE, Narrow-band imaging International Colorectal Endoscopic. 
Table 2 Clinicopathological features

\begin{tabular}{|c|c|}
\hline Feature & \\
\hline Patients, $\mathrm{n}$ & 94 \\
\hline Sex, male: female, $n: n$ & $32: 11$ \\
\hline Age, years, mean (range) & $54.2(42-75)$ \\
\hline \multicolumn{2}{|l|}{ Polyps } \\
\hline Size, mm, mean (range) & $17(2-25)$ \\
\hline Shape & $\begin{array}{l}\text { Pedunculated }(n=7) \text {; sessile } \\
(n=63) ; \text { flat }(n=24)\end{array}$ \\
\hline \multicolumn{2}{|l|}{ Histology, n } \\
\hline Nonneoplastic & $\begin{array}{l}37 \text { (Hyperplastic }[n=32] \text {; sessile } \\
\text { serrated adenoma/polyp }[n=5] \text { ) }\end{array}$ \\
\hline Adenoma & $\begin{array}{l}56 \text { (Low-grade dysplasia }[\mathrm{n}=5 \mathrm{I}] \text {; } \\
\text { high-grade dysplasia }[\mathrm{n}=5] \text { ) }\end{array}$ \\
\hline Invasive cancer & I \\
\hline
\end{tabular}

of lesions. The LCI system used in the present study is an additional image processing technique that enhances the separation of red colors to clearly depict deeper shades of red and to brighten white colors. Recently, Suzuki et al ${ }^{8}$ reported an LCI high-magnification endoscopy method, combined with crystal violet staining, to yield images that closely approximate histopathological findings. This is expected to improve the accuracy of endoscopic assessment of the depth of invasion for early-stage colorectal cancer. The LCI system can be used without optical magnification and is an effective adjunct in daily practice. Although there are several methods for the pathological prediction of colon polyps, the NICE classification system is the only method that can be used without optical magnification.

The results of this study demonstrated the sensitivity, specificity, PPV, and NPV of classifying neoplastic lesions according to the NICE system using LCI data. Rex ${ }^{7}$ recruited 451 consecutive colorectal polyps and reported high-confidence predictions of adenoma and hyperplastic histology (correct for $91 \%$ and $95 \%$, respectively) using NBI without optical magnification. Kim et $\mathrm{al}^{9}$ reported a sensitivity, specificity, PPV, and NPV for neoplastic lesion prediction of $97.5 \%, 85.1 \%, 91.7 \%$, and $95.2 \%$, respectively, using the NBI system without optical magnification in

Table 3 Relationship between NICE classification system and histological findings using $\mathrm{LCl}$ data

\begin{tabular}{|c|c|c|c|c|}
\hline $\begin{array}{l}\text { NICE } \\
\text { classification }\end{array}$ & HP \& SSA/P & LGD & HGD & Carcinoma \\
\hline Type I & 31 (93.9) & $2(6.1)$ & & \\
\hline Type 2 & $6(10.1)$ & $49(83.1)$ & $4(6.7)$ & \\
\hline Type 3 & & & I $(50.0)$ & I $(50.0)$ \\
\hline
\end{tabular}

Note: Data presented as $n$ (\%).

Abbreviations: NICE, Narrow-band Imaging International Colorectal Endoscopic; $\mathrm{LCl}$, linked-color imaging; HP, hyperplastic polyp; SSA/P, sessile serrated adenoma/ polyp; LGD, low-grade dysplasia; HGD, high-grade dysplasia.
Table 4 Performance characteristics of $\mathrm{LCl}$ in predicting neoplastic lesions

\begin{tabular}{lllll}
\hline Lesion & Sensitivity & Specificity & PPV & NPV \\
\hline NICE type 2/3 & 96.5 & 83.8 & 90.2 & 93.9 \\
& $(87.5-99.6)$ & $(67.9-93.8)$ & $(79.8-96.3)$ & $(79.8-99.2)$ \\
\hline
\end{tabular}

Note: Data presented as \% ( $95 \%$ confidence interval).

Abbreviations: NICE, Narrow-band imaging International Colorectal Endoscopic; LCl, linked-color imaging; PPV, positive predictive value; NPV, negative predictive value.

126 colorectal polyps. Our results are similar to those of previous studies investigating the application of the NICE classification using an NBI system without optical magnification. LCI combined with the NICE classification system is a powerful tool for prediction of probable histology of colon polyps. In addition, a recent study showed that LCI improved polyp visibility for both expert and nonexpert endoscopists and was also found to be useful for improving polyp visibility in any location and with any size, morphology, histology, and preparation level. ${ }^{10}$

\section{Limitation}

The major limitation of this study was the small number of polyps assessed. Moreover, most of the patients were referred from a health management center and had no clinical symptoms. The proportion of advanced polyps or invasive cancers found in this study was lower than that found in previous studies. Thus, definitive conclusions cannot be drawn from these data and larger studies are warranted.

In a study by Rex, ${ }^{7}$ large polyps $(>10 \mathrm{~mm})$ had better pathological prediction rates compared with small polyps $(<10 \mathrm{~mm})$. Because we placed no restrictions on the size of polyps included in our study (most were $<10 \mathrm{~mm}$ in size), polyp size may have influenced our pathological prediction rates.

\section{Conclusion}

In conclusion, LCI combined with the NICE classification system is a powerful tool for predicting probable histology of colon polyps. LCI appears to be a convenient and practical tool, even without optimal magnification. Moreover, it maintains natural color tones; therefore, it could replace the need for white light endoscopy during the entire procedure.

\section{Disclosure}

The authors report no conflicts of interest in this work.

\section{References}

1. Zauber AG, Winawer SJ, O'Brien MJ, et al. Colonoscopic polypectomy and long-term prevention of colorectal-cancer deaths. $N$ Engl J Med. 2012;366(8):687-696. 
2. Tanaka S, Sano Y. Aim to unify the narrow band imaging (NBI) magnifying classification for colorectal tumors: current status in Japan from a summary of the consensus symposium in the 79th annual meeting of the Japan Gastroenterological Endoscopy Society. Dig Endosc. 2011; 23 (Suppl 1):131-139.

3. Pigo F, Bertani H, Manno M, et al. i-Scan high-definition white light endoscopy and colorectal polyps: prediction of histology, interobserver and intraobserver agreement. Int J Colorectal Dis. 2013;28(3): 399-406.

4. The Paris endoscopic classification of superficial neoplastic lesions: esophagus, stomach, and colon: November 30 to December 1, 2002. Gastrointest Endosc. 2003;58 (Suppl 6):S3-S43.

5. Morson B. President's address. The polyp-cancer sequence in the large bowel. Proc R Soc Med. 1974;67(6 Pt 1):451-457.

6. Ignjatovic A, East JE, Suzuki N, Vance M, Guenther T, Saunders BP. Optical diagnosis of small colorectal polyps at routine colonoscopy (Detect InSpect ChAracterise Resect and Discard; DISCARD trial): a prospective cohort study. Lancet Oncol. 2009;10(12):1171-1178.
7. Rex DK. Narrow-band imaging without optical magnification for histologic analysis of colorectal polyps. Gastroenterology. 2009;136(4): 1174-1181.

8. Suzuki T, Hara T, Kitagawa Y, Yamaguchi T. Magnified endoscopic observation of early colorectal cancer by linked color imaging with crystal violet staining (with video). Gastrointest Endosc. 2016;84(4): 726-729.

9. Kim JJ, Hong KS, Kim JS, Jung HC. A Randomized Controlled Clinical Study comparing the diagnostic accuracy of the histologic prediction for colorectal polyps depending on the use of either magnified or nonmagnified narrow band imaging. Clin Endosc. 2015;48(6):528-533.

10. Yoshida N, Naito Y, Murakami T, et al. Linked color imaging improves the visibility of colorectal polyps: a video study. Endosc Int Open. 2017; 5(6):E518-E525.
Therapeutics and Clinical Risk Management

\section{Publish your work in this journal}

Therapeutics and Clinical Risk Management is an international, peerreviewed journal of clinical therapeutics and risk management, focusing on concise rapid reporting of clinical studies in all therapeutic areas, outcomes, safety, and programs for the effective, safe, and sustained use of medicines. This journal is indexed on PubMed Central, CAS,

\section{Dovepress}

EMBase, Scopus and the Elsevier Bibliographic databases. The manuscript management system is completely online and includes a very quick and fair peer-review system, which is all easy to use. Visit http://www.dovepress.com/testimonials.php to read real quotes from published authors.

Submit your manuscript here: http://www.dovepress.com/therapeutics-and-clinical-risk-management-journal 